\title{
Magnitude, Severity, and Associated Factors of Anemia among Under-Five Children Attending Hawassa University Teaching and Referral Hospital, Hawassa, Southern Ethiopia, 2016
}

\author{
Yeshimebet Gebereselassie,, ${ }^{1}$ Mesganaw BirhanSelassie, ${ }^{2}$ Tadesse Menjetta $\mathbb{D}^{\mathbb{D}}{ }^{2}$ \\ Jemal Alemu, ${ }^{3}$ and Aster Tsegaye ${ }^{3}$ \\ ${ }^{1}$ Hawassa College of Health Science, Department of Medical Laboratory Science, Hawassa, Ethiopia \\ ${ }^{2}$ Hawassa University, College of Medicine and Health Science, Hawassa, Ethiopia \\ ${ }^{3}$ Department of Medical Laboratory Sciences, School of Allied Health Science, College of Health Science, Addis Ababa University, \\ Addis Ababa, Ethiopia
}

Correspondence should be addressed to Tadesse Menjetta; tademen2000@yahoo.com

Received 11 May 2020; Accepted 29 June 2020; Published 7 August 2020

Academic Editor: Duran Canatan

Copyright (c) 2020 Yeshimebet Gebereselassie et al. This is an open access article distributed under the Creative Commons Attribution License, which permits unrestricted use, distribution, and reproduction in any medium, provided the original work is properly cited.

\begin{abstract}
Background. Anemia is a widespread public health problem associated with increased risk of morbidity and mortality. Infants, under-5-year-old children, and pregnant women have greater susceptibility to anemia. The magnitude and associated risk factors for anemia vary in different settings. The study aimed to assess the magnitude, severity, and associated factors of anemia at Hawassa University Teaching and Referral hospital, Hawassa, southern Ethiopia. Methods. In a hospital-based cross-sectional study, a total of 422 under-five children were included. Sociodemographic data and other predisposing factors were collected by structured questionnaire. Venous blood samples were collected and analyzed for hemoglobin determination using a Cell-Dyn 1800 automated analyzer. Stool samples were collected and processed using direct wet mount and formol-ether concentration method to detect intestinal parasites. Data were entered and analyzed using SPSS version 20 statistical packages. Binary and multiple logistic regressions were computed to assess factors associated with anemia. $p$ value less than 0.05 was taken as statistically significant. Result. The overall prevalence of anemia was found to be $41.7 \%$. The mean hemoglobin level was $10.59 \mathrm{~g} / \mathrm{dl}$. Anemia was of mild, moderate, and severe type in $6.6 \%, 19 \%$, and $16.1 \%$ of the children, respectively. Children in the age group 6-23 months $(\mathrm{AOR}=2.04$ (95\% CI: 1.13, 3.69)), and mothers having no formal education $(\mathrm{AOR}=1.73(95 \% \mathrm{CI}: 0.99,3.02))$ were identified as associated factors for anemia. Conclusion. The prevalence of anemia among the study subjects was $41.7 \%$ indicative of the fact that anemia is an important public health problem. It was associated with the child's age, residence, mother's education level, and intestinal parasite (Ascaris lumbricoides). It clearly indicates that there should be well integrated public health interventions to improve the health status that needs to be prioritized to prevent anemia among children under five years of age.
\end{abstract}

\section{Background}

World Health Organization defines anemia as a decrease in the concentration of circulating red blood cells or in the hemoglobin concentration and a related impaired capacity to transport oxygen. It is also defined as hemoglobin level below $11 \mathrm{mg} / \mathrm{dl}$ for children of 6-59 months [1].

Anemia is classified as microcytic, normocytic, or macrocytic, based on the mean corpuscular volume [2]. It can also be classified based on haemoglobinization of red cells as normochromic, describing normal staining of red cells, as seen when haemoglobinization is adequate, and hypochromic, describing pale staining of red cells, as seen when haemoglobinization is inadequate. Hypochromic cells show an increased area of central pallor [3].

Globally, anemia affects 1.62 billion people, which corresponds to $24.8 \%$ of the population. The highest prevalence is in preschool-age children which is $47.4 \%$. World 
Health Organization regional estimates generated for preschool-age children and pregnant and nonpregnant women indicate that the highest proportion of individuals affected are in Africa (47.5-67.6\%), while the greatest number is in South East Asia where 315 million individuals are affected [1].

World Health Organization showed that 818 million children under the age of five and women are affected by anemia, mainly in developing countries. About one million of them die every year [1]. In the developing world, $42 \%$ of children less than five years of age and $53 \%$ of children $5-14$ years of age are anemic [4]. About $67.6 \%$ of under-five children in Africa are suffering from anemia indicating anemia as a severe health problem. In East Africa, it is estimated that three quarters of under-five children suffer from anemia [1]. Studies conducted in East Africa have shown that $P$. falciparum malaria and iron deficiency account for much of the anemia seen in young children [5].

In Ethiopia, according to the demographic and health survey findings in 2011, more than four out of ten under-five children (44\%) were anemic. From these, about $21 \%$ of children were mildly anemic, $20 \%$ were moderately anemic, and $3 \%$ were severely anemic [6]. The etiology of anemia involves the interaction between multiple factors including nutritional deficiencies, genetic red blood cell disorders, and infectious diseases, particularly malaria and hookworm infections. Human immunodeficiency virus/acquired immunodeficiency syndrome should also increasingly be considered as a direct and indirect contributor to anemia in young age group [7-9].

Like other developing countries, the prevalence of intestinal parasites is widely spread in Ethiopia that can cause anemia in under-five children. Among the common intestinal protozoan parasites, Giardia, Cryptosporidium, and helminths such as Ascaris are widely distributed, where there is overcrowding, poor environmental sanitation, limited economic resources, and poor personal hygienic practice which are predisposing factors. Most of the intestinal parasites are more severe in children than adults, which is associated with malnutrition, growth retardation, and poor care for children. The case is worth in under-five-year-old children because of poor maternal hygiene, play habitats of children in the house in close proximity to one another that creates an appropriate condition for the transmission and spread of the disease [7].

A consequence of anemia may lead to impaired cognitive function, growth, and psychomotor development, especially in children. Infants, under-5-year-old children, and pregnant women have greater susceptibility to anemia because of their increased iron requirements due to rapid body growth and expansion of red blood cells [9-11]. The frequency of anemia in a population may be the most important single indicator of the overall health status of a population regarding malaria, geohelminths infection, and mal- or undernutrition [9].

Anemia in preschool children has a negative effect on cognition, motor development and growth, academic performance, immunity, and susceptibility to infections [12]. Despite these, detailed investigation of anemia in under-five children is limited in Ethiopia and existing data revealed variable magnitude. Therefore, this study is designed to assess the magnitude of anemia and its associated factors among under-5 children attending Hawassa University Teaching and Referral hospital in Hawassa, southern Ethiopia.

\section{Methods}

2.1. Study Area. The study was conducted at Hawassa University Teaching and Referral hospital, located in the city of Hawassa, southern Ethiopia. Hawassa city is found about $270 \mathrm{~km}$ south of the capital Addis Ababa.

2.2. Study Design. A cross-sectional hospital-based study was conducted to determine the magnitude of anemia and associated factors among under-5 children at Hawassa city, southern Ethiopia.

2.3. Study Subjects. All under-five children who were visiting the hospital during the study period and whose parents consented to participate in the study were included. Before data collection, written informed consent was obtained from each parent/caregiver of the study participants after explaining the purpose of the study.

2.4. Data Collection. Semistructured questionnaire was used to collect information such as patients' age, sex, residence, presence of malaria parasite, presence of intestinal parasites, mother's age, and mother's education. Data about the number of children less than 5 years were obtained from their parents/guardians. The tool was prepared after reviewing different related literatures inside and outside the country. The original questionnaire which was prepared in English language was translated into Amharic version and used by trained nurses for data collection. In addition, blood and stool samples for laboratory analyses were collected and analyzed by experienced laboratory professionals.

2.5. Sample Size and Sampling Technique. The minimum sample size required for analysis was calculated using the 95\% confidence interval with 5\% marginal error, using the formula $n=z^{2} p(1-p) / d^{2}$, where $n=$ sample size, $z=$ statistic for a level of confidence $(z=1.96$ at $95 \% \mathrm{CI}), p=$ expected prevalence or proportion $(p=0.50)$, and $d=$ precision (if $5 \%$, $d=0.05$ ), and the sample size was 384 . Allowing $10 \%$ for nonresponse, the final sample size was 422 . A convenient sampling technique was used to select under-five children who fulfilled inclusion criteria during the study period.

2.6. Blood Sample Collection. About $3 \mathrm{ml}$ of venous blood samples were collected by strictly following standard operating procedure. The blood was processed for hemoglobin test. 
2.7. Stool Specimen Collection and Processing. About $1 \mathrm{gm}$ (pea size) fresh fecal specimen was collected using a labeled, clean, dry, leak-proof container [13]. The stool specimen was processed by the wet mount and formol-ether concentration technique.

2.8. Data Management and Statistical Analysis. All questionnaires were checked for completeness. Completed data were coded, entered, cleaned, and analyzed using Statistical Package for Social Sciences version 23. Descriptive statistics were used to summarize continuous variables and frequencies tables were computed to show the distribution of the sociodemographic and clinical characteristics of the patients. To assess associations between risk factors and anemia, bivariate and multivariate logistic regression analyses were used. Odds ratio with $95 \%$ confidence level was used to determine the strength of association. Statistical significance was declared at $p$ value $<0.05$.

2.9. Classification of Anemia. The definition of anemia and classification of severity of anemia were determined based on the hemoglobin value in accordance with the value stated by the World Health Organization [14].

2.10. Ethical Considerations. Ethical clearance was obtained from the ethical review board of Addis Ababa University College of Health Science, School of Allied Health Science, Department of Medical Laboratory Sciences and Institutional Review Board of the College of Medicine and Health Science of Hawassa University. Before data collection, written informed consent was obtained from each parent/ caregiver of the study participants after explaining the purpose of the study.

\section{Results}

3.1. Characteristics of Study Participants. A total of 422 under-five children participated in the study. Of the participants, 235 (55.7\%) were males and 187 (44.3\%) females. The age ranged from 6 to 59 months with mean age of 27.9 $( \pm 16.72)$ months. Regarding their age distribution, 174 (41.2\%) were between 6 and 23 months, 98 (23.2\%) were between 24 and 35 months, 75 (17.8\%) were between 36 and 47 months, and 75 (17.8\%) were between 48-59 months (Table 1).

3.2. Parental Characteristics. All of the parents/guardians were female with mean age of $28.8 \pm 5.0$ years. Regarding the educational status, $119(28.2 \%)$ had attained secondary education and above, $112(26.5 \%)$ were at primary level, and 191 (45.3\%) had no formal education (Table 1).

3.3. Prevalence of Anemia. The overall prevalence of anemia as defined by hemoglobin levels lower than $11 \mathrm{~g} / \mathrm{dl}$ was found to be $41.7 \%(176 / 422)$. Of them, $44.3 \%$ (78/176) were females and $55.7 \%$ (98/176) were males. The frequency of anemia declined with age; it was highest in the youngest age
TABLE 1: Sociodemographic characteristics of children aged 6-59 months and their parents/guardians attending Hawassa University Teaching and Referral hospital, Hawassa, southern Ethiopia, 2016.

\begin{tabular}{|c|c|c|}
\hline$\underline{\text { Parameters }}$ & Frequency & Percentage \\
\hline \multicolumn{3}{|l|}{ Age of child (in months) } \\
\hline $6-23$ & 174 & 41.2 \\
\hline $24-35$ & 98 & 23.2 \\
\hline $36-47$ & 75 & 17.8 \\
\hline $48-59$ & 75 & 17.8 \\
\hline \multicolumn{3}{|l|}{ Sex of child } \\
\hline Male & 235 & 55.7 \\
\hline Female & 187 & 44.3 \\
\hline \multicolumn{3}{|c|}{ Parents/guardians characteristics } \\
\hline Urban & 284 & 67.3 \\
\hline Rural & 138 & 32.7 \\
\hline \multicolumn{3}{|l|}{ Age group (in year) } \\
\hline$<30$ & 241 & 57.1 \\
\hline$\geq 30$ & 181 & 42.9 \\
\hline \multicolumn{3}{|l|}{ Educational status } \\
\hline No formal education & 191 & 45.3 \\
\hline Primary & 112 & 26.5 \\
\hline Secondary and above & 119 & 28.2 \\
\hline \multicolumn{3}{|l|}{ Occupation } \\
\hline Housewife & 140 & 33.2 \\
\hline Employed & 116 & 27.5 \\
\hline Small scale business & 79 & 18.7 \\
\hline Farmer & 80 & 19.0 \\
\hline Others & 7 & 1.6 \\
\hline
\end{tabular}

group of less than 2 years (48.9\%) and lowest (34.7\%) in the oldest age group.

Regarding the severity of anemia, among sampled children, 68 (16.1\%) of them were severely anemic, whereas $80(19 \%)$ were moderately anemic and $28(6.6 \%)$ were mildly anemic (Table 2).

3.4. Factors Associated with Anemia. Regarding the result of intestinal parasites among study subjects, overall, 77 (18.2\%) were infected with intestinal parasites. As shown in Table 3, Ascaris lumbricoides accounted for the highest 47 (11.1\%) followed by E. histolytica 18 (4.3\%), hookworm 17 (4.0\%), and G. lamblia $3(0.7 \%)$. The prevalence was $44(18.7 \%)$ for male and $33(17.6 \%)$ for female. It was high among the age group of $48-59$ months (32\%) compared to other age groups.

In bivariate (unadjusted) logistic regression analysis, statistically significant associated factors of anemia were children aged 6-23 months, rural residence, children whose mothers had no formal education, and infection with Ascaris lumbricoides. However, in multivariate (adjusted) logistic regression analysis, statistically significant associated factors of anemia were only children aged 6-23 months while children whose mothers had no formal education had a borderline statistical significance.

Children aged 6-23 months had two times odds of being anemic than those aged 48-59 months (AOR $=2.0$ (95\% CI: $1.13,3.69) ; p$ value 0.017 ). The odds of being anemic among children whose mothers had no formal education were 
TABLE 2: Distribution of hemoglobin level among male and female under-5 children at Hawassa University Teaching and Referral hospital, Hawassa, southern Ethiopia, 2016.

\begin{tabular}{lccc}
\hline Hgb value & Male (\%) & Female (\%) & Total (\%) \\
\hline$<7$ g/dl (severe) & $40(17.0)$ & $28(15.0)$ & $68(16.1)$ \\
$7-9.9$ g/dl (moderate) & $40(17.0)$ & $40(21.4)$ & $80(19.0)$ \\
$10-10.9$ g/dl (mild) & $18(7.7)$ & $10(5.3)$ & $28(6.6)$ \\
$\geq 11 \mathrm{~g} / \mathrm{dl}$ (nonanemic) & $137(58.3)$ & $109(58.3)$ & $246(58.3)$ \\
\hline
\end{tabular}

TABLE 3: Frequency of intestinal parasites among under-5 children in Hawassa University Teaching and Referral hospital, Hawassa, southern Ethiopia, 2016.

\begin{tabular}{|c|c|c|}
\hline Species & $\begin{array}{c}\text { Positive } \\
N(\%)\end{array}$ & $\begin{array}{c}\text { Negative } \\
N(\%)\end{array}$ \\
\hline Ascaris lumbricoides & $47(11.1)$ & $375(88.9)$ \\
\hline Hookworm & $17(4.0)$ & $405(96.0)$ \\
\hline Entamoeba histolytica & $18(4.3)$ & $404(95.7)$ \\
\hline Giardia lamblia & $3(0.7)$ & $419(99.3)$ \\
\hline
\end{tabular}

TABLE 4: Multivariate logistic regressions of selected variables associated with anemia among under-five children in Hawassa University Teaching and Referral hospital, southern Ethiopia, 2016.

\begin{tabular}{|c|c|c|c|c|c|}
\hline \multirow{2}{*}{ Risk factor } & \multirow{2}{*}{$\begin{array}{c}\text { Anemia } \\
N(\%)\end{array}$} & \multicolumn{2}{|c|}{ Unadjusted (COR) } & \multicolumn{2}{|c|}{ Adjusted (AOR) } \\
\hline & & OR $(95 \% \mathrm{CI})$ & $p$ value & OR $(95 \% \mathrm{CI})$ & $p$ value \\
\hline \multicolumn{6}{|l|}{ Child age } \\
\hline $6-23$ & $85(48.9)$ & $1.8[1.0-3.1]$ & 0.040 & $2.04[1.13-3.69]$ & 0.017 \\
\hline $24-35$ & $39(39.8)$ & $1.2[0.7-2.3]$ & 0.490 & $1.52[0.78-2.91]$ & 0.211 \\
\hline $36-47$ & $26(34.7)$ & $1[0.5-1.9]$ & 1.000 & $1.11[0.55-2.24]$ & 0.759 \\
\hline 48-59 & $26(34.7)$ & 1 & & 1 & \\
\hline \multicolumn{6}{|l|}{ Residence } \\
\hline Urban & $109(38.4)$ & 1 & & 1 & \\
\hline Rural & $67(48.6)$ & $1.5[1.0-2.3]$ & 0.047 & $1.24[0.76-2.02]$ & 0.378 \\
\hline \multicolumn{6}{|l|}{ Mother's education level } \\
\hline No formal & $62(48.1)$ & $1.67[1.0-2.7]$ & 0.033 & $1.74[1.00-3.02]$ & 0.051 \\
\hline Primary & $57(42.9)$ & $1.4[0.8-2.2]$ & 0.207 & $1.43[0.82-2.52$ & 0.208 \\
\hline Secondary or above & $57(35.6)$ & 1 & & 1 & \\
\hline \multicolumn{6}{|l|}{ A. lumbricoides } \\
\hline Yes & $29(61.7)$ & $2.5[1.3-4.7]$ & 0.004 & $2.44[0.93-6.46]$ & 0.071 \\
\hline No & $147(39.2)$ & 1 & & 1 & \\
\hline
\end{tabular}

nearly two times more compared to those children whose mothers had secondary and above education level $(\mathrm{AOR}=1.7$ (95\% CI: 1.0-3.0); $p$ value 0.051). However, this finding was with borderline statistical significance in multivariate but was statistically significant in bivariate analysis (Table 4). In bivariate (unadjusted) logistic regression analysis, children living in rural area had 1.2 times odds of being compared to their urban counterparts $(\mathrm{AOR}=1.5$ (95\% CI: 1.0-2.3); $p$ value 0.047). Furthermore, in bivariate (unadjusted) logistic regression analysis, children who were infected with Ascaris lumbricoides had 2.5 times odds of being anemic than those who were not $(\mathrm{AOR}=2.5$ (95\% CI: 1.3-4.7); $p$ value 0.004).

\section{Discussion}

This study showed that the overall prevalence of anemia among under-five children was $41.7 \%$ and the mild, moderate, and severe anemia were $6.6 \%, 19 \%$, and $16.1 \%$, respectively. The prevalence of anemia in this study is comparable with findings in Bangladesh (40.9\%) conducted in 2010 [15], North Kazakhstan (40.0\%) [16], and national prevalence EDHS 2011 (44\%) [6]. However, the finding is lower than that of estimated global anemia prevalence (47.4\%) [1] and those reported from Ghana (78.4\%) in 2014 [17], Cape Verde (51.8\%) in 2014 [18], northern part of Ethiopia (50.3\%) [19], Nigeria (70.5\%) in 2012 [20], Tanzania (77.2\%) in 2015 [21], and Kenya (71.8\%) in 2005 [11]. The lower prevalence in this study might be due to the change made by the existing nutritional, public health interventions, easy accessibility of health information through health extension workers, and among other factors that may be included. But it is higher than the study conducted in Eastern Cuba in 2011 which is 26\% [22], 28.8\% from Kenya in 2014 [10], and 38.8\% from Haiti 2013 [23]. The possible reason for these differences may be due to geographical and seasonal differences, interventions used, lifestyles, and socioeconomic status. 
In this study, the magnitude of severe anemia among the study participants was $16.1 \%$. This result is lower than the study conducted in Tanzania (27.7\%) [21]. But it was higher than the findings in western China which documented 3.2\% severe anemia [24]. In this study, the severity of anemia significantly associated with residence; children living in rural area were highly susceptible to severe anemia. A similar finding was reported in Tanzania [25].

In the present study, children who were less than 23 months were found more likely to be anemic. This finding is similar to studies conducted in southern Cameroon in 1998 [25], northern Ethiopia in 2014 [26], and Tanzania in 2015 [21]. Children of mothers with no formal education were more anemic than those with mothers having secondary and above level of education. Educated mothers are more conscious of their children's health and introducing scientifically proven feeding practices, which help to improve their children's nutritional status [27]. This result is comparable with studies conducted in northeast Ethiopia (2015) [28], Burma (2012) [29], Tanzania (2015) [21], Brazil (2013) [30], and India (2014) [12].

In this study, in bivariate logistic regression analysis, children who were infected with Ascaris lumbricoides infection were more likely to be anemic. This result was supported by reports from Nigeria [31] and Malaysia [32], but studies conducted in Indonesia [33] and Senegal [34] show no association between Ascaris lumbricoides infection and anemia.

\section{Conclusion}

The prevalence of anemia among the study subjects was $41.7 \%$ indicative of the fact that anemia is an important public health problem. It was associated with the child's age, residence, mother's education level, and intestinal parasite (Ascaris lumbricoides). It indicates that there should be well integrated public health interventions to improve the health status that needs to be prioritized to prevent anemia among children under five years of age.

\section{Data Availability}

The datasets used and/or analyzed during the current study are available from the corresponding author on reasonable request.

\section{Additional Points}

Study Limitations. In this study, only anemia was measured, the inclusion of other measures such as serum ferritin would help to measure the status of iron.

\section{Ethical Approval}

Ethical clearance was obtained from the Institutional Review Board (IRB) of Hawassa University College of Medicine and Health Sciences.

\section{Conflicts of Interest}

All authors have declared that no conflicts of interest exist.

\section{Authors' Contributions}

YG was responsible for the conception of the research idea, study design, and proposal development. MB collected and ensured the quality of the data. AT and JA have supervised the overall activities. TM analyzed and interpreted the data. All authors critically reviewed the manuscript. All authors gave final approval of the paper to be published. TM, the corresponding author, had the responsibility to submit the manuscript for publication.

\section{Acknowledgments}

The authors would like to acknowledge Addis Ababa University, College of Health Science, Department of Medical Laboratory Sciences. The authors also appreciate the management and staff of Hawassa University College of Medicine and Health Science, Referral Hospital, for their encouragement and support. They also acknowledge the study participants for their willingness to participate in the study.

\section{References}

[1] E. Mclean, M. Cogswell, I. Egli, D. Wojdyla, and B. de Benoist, "Worldwide prevalence of anaemia, WHO vitamin and mineral nutrition information system, 1993-2005," Public Health Nutrition, vol. 12, no. 4, pp. 444-454, 2008.

[2] H. Ferry and W. Virginia, "Evaluation of anemia in children," American Family Physician, vol. 81, no. 12, pp. 1462-1471, 2010.

[3] M. Cheesbrough, District Laboratory Practice in Tropical Countries Part 2, Cambridge University, Cambridge, UK, 2nd edition, 2006.

[4] K. Tolentino and J. F. Friedman, "An update on anemia in less developed countries," The American Journal of Tropical Medicine and Hygiene, vol. 77, no. 1, pp. 44-51, 2007.

[5] D. Schellenberg, J. R. Schellenberg, A. Mushi et al., "The silent burden of anaemia in Tanzanian children: a communitybased study," Bulletin of the World Health Organization, vol. 81, no. 8, pp. 581-590, 2003.

[6] Ethiopia Central Statistical Agency and ICF International, $I$. Demographic and Health Survey: Key Findings. Calverton, Maryland UC, 2011, and II. Ethiopia Demographic and Health Survey Key Findings, Ethiopia Central Statistical Agency, Addis Ababa, Ethiopia, 2011.

[7] Y. Aleka, G. Seife, W. Tamir, M. Birhane, and A. Alemu, "Prevalence and associated risk factors of intestinal parasitic infection among under five children in university of Gondar hospital, Gondar, northwest Ethiopia," Biomedical Research and Therapy, vol. 2, no. 8, pp. 347-353, 2015.

[8] R. Ferreira, "Prevalence of anemia in under five-year-old children in a children's hospital in Recife, Brazil," Revista Brasileira de Hematologia e Hemoterapia, vol. 33, no. 2, pp. 100-104, 2010.

[9] D. J. Richardson, K. R. Richardson, K. E. Richardson et al., "Malaria, intestinal parasitic infection, anemia, and malnourishment in rural Cameroonian villages with an 
assessment of early interventions," Journal of the Arkansas Academy of Science, vol. 65, pp. 72-97, 2011.

[10] O. Ngesa and H. Mwambi, "Prevalence and risk factors of anaemia among children aged between 6 months and 14 years in Kenya," PLoS One, vol. 9, no. 11, pp. 1-10, 2014.

[11] M. R. Desai, P. A. Phillips-howard, D. J. Terlouw et al., "Factors associated with hemoglobin concentrations in preschool children in western Kenya: cross-sectional studies," The American Journal of Tropical Medicine and Hygiene, vol. 72, no. 1, pp. 47-59, 2005.

[12] A. Baranwal, A. Baranwal, and N. Roy, "Association of household environment and prevalence of anemia among children under-5 in India," Frontiers in Public Health, vol. 2, pp. 1-7, 2014.

[13] M. Cheesbrough, District Laboratory Practice in Tropical Countries, Part 1, Cambridge University Press, Cambridge, UK, 2nd edition, 2009.

[14] WHO, Haemoglobin Concentrations for the Diagnosis of Anaemia and Assessment of Severity, Department of Nutrition for Health and Development (NHD), WHO, Geneva, Switzerland, 2011.

[15] M. K. Uddin, M. H. Sardar, M. Z. Hossain et al., "Prevalence of anaemia in children of 6 months to 59 months in Narayanganj, Bangladesh," Journal of Dhaka Medical College, vol. 19, no. 2, pp. 126-130, 2010.

[16] S. Tazhibayev, O. Dolmatova, Z. Yessimsiitova et al., "Prevalence of anaemia in under five-years-old children in three counties of Kazakhstan: a cross-sectional study," European Journal of Public Health, vol. 24, no. 2, pp. 272-275, 2014.

[17] J. E. Ewusie, C. Ahiadeke, J. Beyene, and J. S. Hamid, "Prevalence of anemia among under-5 children in the Ghanaian population: estimates from the Ghana demographic and health survey," BMC Public Health, vol. 626, no. 14, pp. 1-9, 2014.

[18] R. M. L. Semedo, M. M. A. S. Santos, M. R. Baião, R. R. Luiz, and G. V. Veiga, "Prevalence of anaemia and associated factors among children below five years of age in Cape Verde, West Africa," Journal of Health, Population, and Nutrition, vol. 32, no. 4, pp. 646-657, 2014.

[19] D. Habte, K. Asrat, M. G. Magafu et al., "Maternal risk factors for childhood anaemia in Ethiopia," African Journal of Reproductive Health, vol. 17, no. 3, pp. 110-118, 2013.

[20] V. U. Muoneke, R. C. Ibekwe, H. U. Nebe-Agumadu, and B. C. Ibe, "Factors associated with mortality in under-five children with severe anemia in Ebonyi, Nigeria," Indian Pediatrics, vol. 49, no. 2, pp. 119-123, 2012.

[21] R. H. Simbauranga, E. Kamugisha, A. Hokororo, B. R. Kidenya, and J. Makani, "Prevalence and factors associated with severe anaemia amongst under-five children hospitalized at Bugando medical centre, Mwanza, Tanzania," BMC Hematology, vol. 15, no. 1, pp. 1-9, 2015.

[22] K. Paulí, M. Leyva, C. Arocha, and D. Herrera, "Anemia in children under five years old in eastern Cuba, 2005-2011," MEDICC Review, vol. 16, no. 1, pp. 16-23, 2014.

[23] M. A. Ayoya, I. Ngnie-Teta, M. N. Séraphin et al., "Prevalence and risk factors of anemia among children 6-59 months old in Haiti," Anemia, vol. 2013, Article ID 502968, 3 pages, 2013.

[24] W. Gao, H. Yan, S. Dang, and L. Pei, "Severity of anemia among children under 36 months old in rural western China," PLoS One, vol. 8, no. 4, pp. 2-7, 2013.

[25] M. Cornet, M. Cot, N. Fievet et al., "Prevalence of and risk factors for anemia in young children in southern Cameroon," The American Journal of Tropical Medicine and Hygiene, vol. 58, no. 5, pp. 606-611, 1998.
[26] G. Gebreegziabiher, B. Etana, and D. Niggusie, "Determinants of anemia among children aged 6-59 months living in Kilte Awulaelo Woreda, northern Ethiopia," Anemia, vol. 2014, Article ID 245870, 9 pages, 2014.

[27] L. P. Israel, M. B. Filho, P. I. C. de Lira, L. N. Figueiroa, and M. M. Osório, "Prevalence of anemia and associated factors in children aged 6-59 months in Pernambuco, northeastern Brazil," Journal of Public Health, vol. 45, no. 3, pp. 457-466, 2011.

[28] H. Woldie, Y. Kebede, and A. Tariku, "Factors associated with anemia among children aged 6-23 months attending growth monitoring at Tsitsika Health Center, Wag-Himra zone, northeast Ethiopia," Journal of Nutrition and Metabolism, vol. 2015, Article ID 928632, 9 pages, 2015.

[29] A. Zhao, Y. Peng, J. Li et al., "Prevalence of anemia and its risk factors among children 6-36 months old in Burma," The American Journal of Tropical Medicine and Hygiene, vol. 87, no. 2, pp. 306-311, 2012.

[30] M. S. Leite, A. M. Cardoso, C. E. Coimbra Jr. et al., "Prevalence of anemia and associated factors among indigenous children in Brazil : results from the First National Survey of Indigenous People's Health and Nutrition," Nutrition Journal, vol. 12 , no. 1, p. $69,2013$.

[31] F. Osazuwa, O. Ayo, and P. Imade, "A significant association between intestinal helminth infection and anaemia burden in children in rural communities of Edo state, Nigeria," North American Journal of Medical Sciences, vol. 3, no. 1, pp. 30-34, 2011.

[32] R. Ngui, Y. A. L. Lim, L. Chong Kin, C. Sek Chuen, and S. Jaffar, "Association between anaemia, iron deficiency anaemia, neglected parasitic infections and socioeconomic factors in rural children of west Malaysia," PLoS Neglected Tropical Diseases, vol. 6, no. 3, Article ID e1550, 2012.

[33] C. Annrie Eidwina, L. Faridah, Y. Setiati Ermaya, and D. Akhmad Gurnida, "Association of ascariasis with nutritional and anemic status in early school-age students, Indonesia," Althea Medical Journal, vol. 3, no. 1, 2016.

[34] R. C. K. Tine, B. Faye, C. T. Ndour et al., "Parasitic infections among children under five years in Senegal: prevalence and effect on anaemia and nutritional status," ISRN Parasitology, vol. 2013, Article ID 272701, 6 pages, 2013. 